\title{
Nouveau droit de la prescription
}

\author{
Ursina Pally Hofmann
}

Dr iur., secrétaire générale et cheffe de la division Service juridique, FMH

\author{
Le nouveau droit de la prescription entrera en vigueur le $1^{\text {er }}$ janvier 2020. Cela n'est \\ pas sans conséquence pour les médecins, en particulier en ce qui concerne l'obliga- \\ tion de conserver les dossiers des patients et la couverture d'assurance après la ces- \\ sation de leur activité professionnelle indépendante. La FMH recommande de \\ conserver désormais les dossiers médicaux pendant vingt ans et de conclure des \\ polices d'assurances prévoyant une couverture encore durant les vingt ans suivant \\ la cessation d'activité.
}

En guise de compromis, tant le Conseil national que le Conseil des Etats ont, dans le cadre de leur vote final du 15 juin 2018, décidé de prolonger le délai de prescription absolu de dix à vingt ans en cas de dommages corporels et de décès [1]. Aucun référendum n'ayant été déposé contre ce nouveau délai, la réglementation entrera en vigueur le $1^{\mathrm{er}}$ janvier 2020. Ce délai s'appliquera dès lors aux médecins exerçant en tant qu'indépendants et, dans certains cas, aux cliniques privées.

\section{Problématique et genèse}

La problématique des délais de prescription trop courts se pose avant tout en cas de dommages différés, par exemple en cas d'exposition à l'amiante. Après le dépôt, en 2007, d'une motion de la Commission des affaires juridiques du Conseil national sur le sujet, «l'arrêt amiante» de la Cour européenne des droits de l'homme a remis la problématique à l'ordre du jour en 2011 [2]. La cour a considéré qu'en cas de dommages différés, les délais de prescription en vigueur en Suisse rendaient impossible la réclamation en justice de dommagesintérêts et d'indemnités pour tort moral.

La FMH est parvenue à la conclusion qu'une prolongation du délai de prescription pour tous les dommages corporels et les décès amènerait plus de risques et d'incertitudes que d'aspects positifs.

Le projet du Conseil fédéral concernant la modification des délais de prescription a tenu compte de cette critique et prévoyait un délai de prescription absolu de trente ans en cas de lésion corporelle et d'homicide [3].
La FMH s'est penchée sur les avantages et les inconvénients de cette révision de la loi et est parvenue à la conclusion qu'une prolongation du délai de prescription pour tous les dommages corporels et les décès amènerait plus de risques et d'incertitudes que d'aspects positifs. La FMH a fait valoir ses arguments dans le cadre de la consultation et au Parlement. Par ailleurs, la "table ronde sur l'amiante» a entrepris des travaux dans le but de trouver une solution pour les victimes de l'amiante et leurs proches qui n'ont pas eu la possibilité de réclamer une indemnisation en raison de la prescription de leur prétention. Ces travaux sont désormais terminés et un Fonds d'indemnisation des victimes de l'amiante (FIVA), qui propose conseils et soutien financier, a été créé, le 28 mars 2017, à l'initiative privée d'associations et d'entreprises [4].

\section{Avantages et inconvénients, portée pratique}

La nouvelle réglementation apportera certaines améliorations réelles à quelques personnes lésées afin de préserver leurs droits. Toutefois, de nombreuses victimes de dommages différés ne seront pas davantage en mesure de faire valoir leurs prétentions dans le délai de vingt ans; les mésothéliomes malins, notamment, se manifestent après un temps de latence moyen de 35 ans [5]. Dès lors, l'utilité de la prolongation est faible, si seule une partie des patients concernés en profite, ce dont le Parlement avait aussi conscience [6]. Le traitement juridique de tous les autres dommages corporels et décès est sans autre possible dans un délai de prescription de dix ans.

Le temps de latence plus long entre l'événement dommageable et l'éventuelle procédure relative à une pré- 
tention en indemnisation rend plus difficile la preuve que doit apporter le patient, ce qui relativise l'utilité d'un délai de prescription plus long. En outre, cela génère une discrépance encore plus grande avec les délais de prescription cantonaux - certains clairement plus courts - qui s'appliquent à certains hôpitaux [7]. Le défaut d'harmonisation dans ce domaine entretient une insécurité juridique.

La portée pratique la plus importante pour les fournisseurs de prestations découle de l'absence d'adaptation de la durée de l'obligation de conserver les dossiers médicaux au délai de prescription [8]. En général, le dossier médical doit être conservé pendant les dix années suivant la fin du dernier traitement. Il peut ensuite être détruit, sauf s'il est prévisible ou déjà clair que le traitement fera l'objet d'un litige juridique. Des indices dans ce sens existent si le patient est insatisfait du traitement et/ou de son état de santé et l'exprime, ou s'il formule plus ou moins expressément des soupçons d'erreur médicale. Si le médecin doit s'attendre à un litige juridique, il ne peut pas détruire le dossier médical sans, d'une part, s'exposer à des sanctions pénales et, d'autre part, s'attendre à ce que sa situation soit péjorée dans le cadre d'une procédure civile.

Si le médecin doit s'attendre à un litige juridique, il ne peut pas détruire le dossier médical.

Certes, la restitution de leur dossier médical à tous les patients à l'échéance d'un délai de dix ans allègerait la tâche du médecin en matière d'archivage. Cependant, en cas de procédure judiciaire ultérieure, la disponibilité du dossier médical échapperait à sa sphère d'influence; c'est pourquoi nous déconseillons de procéder de la sorte.

L'archivage volontaire des dossiers médicaux au moins jusqu'à l'échéance du nouveau délai de prescription est possible, mais avec les contraintes techniques et financières qui y sont liées.

Tant qu'il n'y a pas d'harmonisation entre le délai de conservation et le délai de prescription, le médecin doit lui-même décider dans quels cas il peut détruire sans conséquence les dossiers médicaux à l'échéance du délai de conservation. A l'heure actuelle, les cantons ne sont pas encore en mesure de dire s'ils vont augmenter la durée de l'obligation de conserver les dossiers médicaux à vingt ans. Ils sont toutefois conscients de la problématique, et on peut s'attendre, sur la base des informations communiquées, à ce qu'une adaptation fasse au moins l'objet de discussions. Dans la mesure où, d'une part, on ignore comment un juge apprécierait la destruction - licite - d'un dossier médical au terme du délai de dix ans, et d'autre part, comme il est probable que les délais de conservation cantonaux seront adaptés, nous recommandons aux médecins de conserver les dossiers médicaux pendant vingt ans.

\section{Les cantons ne sont pas encore en mesure} de dire s'ils vont augmenter la durée de l'obligation de conserver les dossiers médicaux à vingt ans.

La prolongation du délai de prescription a également une portée pratique sur la couverture de la police d'assurance responsabilité civile. En cas de changement d'assureur, le nouveau délai ne joue aucun rôle. Les dommages sont traités selon le principe «claims made» sous l'empire de la police active au moment où le dommage est annoncé, indépendamment du moment auquel sa cause est survenue. Il en va différemment en cas de cessation d'une activité professionnelle indépendante. La couverture doit à l'avenir être assurée pendant les vingt ans suivant cette cessation. Les polices existantes doivent être complétées dans ce sens, et les nouvelles polices doivent prévoir une couverture ultérieure de vingt ans à compter de la cessation d'activité.

\section{Références}

1 Texte soumis au référendum: https://www.bj.admin.ch/bj/fr/ home/wirtschaft/gesetzgebung/verjaehrungsfristen.html; droit de la prescription: https://www.parlament.ch/fr/ratsbetrieb/ suche-curia-vista/geschaeft?AffairId=20130100

2 Arrêt de la CEDH: https://hudoc.echr.coe.int/eng\#\{«itemid»: [«001-141567»]\}

3 Documentation sur la législation en matière de délais de prescription: https://www.bj.admin.ch/bj/fr/home/wirtschaft/gesetzgebung/verjaehrungsfristen.html

4 https://www.stiftung-efa.ch/

5 Factsheet: http://www.forum-asbest.ch/fr/gesundheitsrisiken_fa/ asbestbedingte_krankheiten_fa/

6 Débats finaux: https://www.parlament.ch/fr/ratsbetrieb/suchecuria-vista/geschaeft?AffairId=20130100

7 Par ex. la loi sur la responsabilité du canton de Zurich (Haftungsgesetz Kanton Zürich), § 24: https://www.zh.ch/internet/de/rechtliche_grundlagen/gesetze/erlass.html?Open\&Ordnr=170.1

8 Par ex. la loi sur la santé du canton de Zurich (Gesundheitsgesetz Kanton Zürich), § 13 al. 3: https://www.zh.ch/internet/de/rechtliche_grundlagen/gesetze/erlass.html?Open\&Ordnr=810.1 